\title{
TAGGED : DIGITAL PHOTOGRAPHY AND ARCHAEOLOGISTS
}

\author{
Hugh Corley \\ Research Department \\ English Heritage \\ Fort Cumberland \\ Fort Cumberland Road \\ Eastney, Portsmouth \\ UK \\ hugh.corley@english-heritage.org.uk
}

\begin{abstract}
By looking at both the formal and informal dissemination of archaeological photography this paper will examine the way that digital images are shared and recorded. The influence that this technology has had on working practice will be explored. Through the examination of images on Flickr and Facebook issues of user engagement and data quality will be discussed. This will then be compared against the data held within archives. Finally how these two very different image stores can learn from each other will be explored.
\end{abstract}

\section{INTRODUCTION}

This paper intends to examine archaeological photography, the different forms and purposes, the value both as an archive and socially. With increased prominence of social networking sites and greater availability of digital cameras the images that are captured have changed and requires a re-examination of how archaeologists view photography and how archaeologists are using these images within these social networks both formally and informally. Finally, it will be argued that the changing role of archaeological photographs cannot be ignored and that as a profession we need to embrace the social role of many of our photographs but in a responsible manner that ensures the necessary information is retained, to enable as many of our photographs as possible, formal and informal will be available and informative to future generations.

\section{THE FORMAL AND INFORMAL PHOTOGRAPHIC RECORD}

Site photography constitutes part of the overall archaeological record. Archaeological elements are recorded in a systematic way on 'pro forma' to ensure a consistent standard. Detailed scaled drawings are prepared, capturing the different elements and their relationships to each other. Taken in combination with the finds, samples, surveyed points, lines and polygons, photographs are used to interpret the original purpose of the site and the actions of those who exploited it to tell a story about that past. Frequently there is not one piece of evidence that makes a point but the interpretation of all of the data taken as whole that shapes the final analysis. 
EVA 2009 London Conference 6-8 July

Hugh Corley

On the Record

The purpose of formal photography according to English Heritage's Archaeological Projects Recording Manual "is to provide a visual record of projects as they proceed, and show working conditions and progress" [1]. The specific genera of photography that is practised in the field by archaeologists is critical for re-enforcing and filling in gaps and complementing the archaeological record. Like all genres, archaeological photographs have specific, well-rehearsed rules that ensure the required purposes are met and intended messages are conveyed. The emphasis is on an objective perspective, sterile photographs devoid of all tools and ideally all loose soil and trowel markings [2]. The absence of the archaeologist is a persistent component, only appearing by accident, the toe of their boot or their silhouette holding a camera. They are not the point and they are not to be seen.

Of course it is not always possible to avoid capturing archaeologists in photographs. Sometimes they are required to point out a significant element, provide scale or even to hold the scale in place. According to Conlon, "they must not merely lean on a shovel and admire the view. Above all, they should not be looking (or grinning!) at the camera, but doing something" [3]. The expectation appears to be that their presence must provide a benefit to the archaeology.

This expectation may have been shaped by the cost and effort associated with procuring and processing wet film (either black and white or colour slide film). This meant that photographs were precious, only taken to record what was absolutely necessary and part of the archaeological record. Frequently, the final frames were used to capture the landscape, weather and the archaeologists especially at the end of a project. The only consistent exception is the end of excavation group portrait. All the archaeologists lined up for the formal photographs, usually sufficiently dirty and scruffy, occasionally with a dog.

The advent of the digital camera changed this although not as quickly as one might expect. Working practice increased the numbers, types and uses of formal archaeological images. However, they were not always included in the final archive. For example, one of my previous employers was taken aback when a competing archaeology firm sent an email with a digital photograph attached of a particularly interesting discovery to the regional archaeological inspector. This motivated the inspector to desire this sort of immediate feedback from the other archaeological units as well. At the time digital images were still not considered to be of sufficient quality or reliable over the long-term for formal use by the profession (there are still very strong reservations). It is very likely that, although this image was sent to the inspector, it most likely did not ended up in the archive. Where possible digital images were captured and were maintained in the digital record. Being digital, initially these images began appearing on project or commercial unit websites and in PowerPoint presentations but there were still reservations about their appropriateness for the final archive. Greatest of which was the lack of appropriate means of archiving most of these images. Instead the preference was to use digital photography for working shots and particularly interesting highlights, frequently captured by supervisor or director (the only one who could be trusted with such expensive kit). Broadly speaking this was not only reflected in the 
types of images captured but in the lack of supplemental information to describe them. The standard archaeological photograph is recorded with information describing the project, shot and roll number, the direction it was taken from, a clear description of the subject, the date and the initials of the photographer. Far too frequently digital photography was not recorded with the same diligence.

The value of most of these early digital images is questionable, their informal nature more often than not meant that the full set of information required for re-use is absent (such as exact location and date). The absence of this data greatly degrades their perceived value and frequently leads to their absence in the archive. If these images have not ended up on Facebook or Flickr, it is likely they may never see the light of day again.

The formal adoption of digital images within archaeology has been a gradual and reluctant process likely encouraged by the high costs of obtaining and processing wet film and an increased faith in the longevity of the digital version. The reduced cost of digital storage has inevitably played a significant role as well. This gradual adoption has meant that archaeologists frequently owned digital cameras (sometimes in their phones) long before they were provided with one on site.

\section{Off the record}

The digital photographs taken with the archaeologists' personal cameras are rarely captured as part of the digital record and even less so as part of the archive. Instead they appear on social networking sites like Flickr and Facebook. They are not part of the whole record to be scrutinised and aided in interpretation, despite the fact that some of these images may do a better job than those within the formal record. Instead, their value is in the conversations and interactions that occur around them; visitors and friends tagging images, frequently with precisely the information the archive would require.

The informal photography being discussed documents our lives; these are the shots we take attempting to capture memories and to share with friends. The proliferation of digital cameras and camera phones has seen an increase in informal photography by the archaeologists. Unlike formal archaeological photography, these images attempt to capture the highlights of the archaeology not the meticulous details of the excavation process at each stage. Instead, like the early formal digital photographs these informal photographs focus on the really impressive find or interesting archaeological element. The primary purpose is to document the people and the moments you would like share with others. This is not to the exclusion of the archaeology but along side it. In many ways they are diametrically opposed to the formal archaeological photographs in that frequently they aspire to capture dirty people, soaked through with tools in hand giving a dirty look for capturing such an unpleasant or embarrassing moment. Sometimes they even dare to lay down there tools and grin while they lay soaking up the sun. The composition is frequently about the moment that is changing, not a staged photograph intending to show a specific archaeological element. 


\section{GETTING THE WORD OUT}

Now that the different roles, applications, and values of these images have been discussed, it is worth turning our attention to the different paths to the archive and eventual dissemination that the two types of images take. They could not be more different.

Typically, the path for formal archaeological field film photography is for the photograph to be taken and then recorded on a photographic record form. Typically, this form will record roll, frame, date, description, direction and the photographer's initials. These images will then be sent off to be processed. The field archaeologists rarely, if ever, will see these photographs while still in the field. At the conclusion of field work, the project manager will keep these photographs and when required use them to understand relationships and provide a different perspective than is obtained from detailed drawings, site plan and 'pro forma' data [4].

Digital photography has gone some way to change this model. Digital cameras provided immediate feedback and allowing errors to be noticed and photographs to be re-taken where necessary. Where previously the field archaeologist never saw these images, they are able to see them immediately, not only on the camera but increasingly often on site laptops. At the conclusion of fieldwork these digital images are accessible over the computer networks for the entire team to use.

Eventually, most of these film and increasingly digital photographs, along with the scaled drawings and plans, 'pro forma' data, finds and synthesised reports will be deposited as an archive and disseminated. The Institute For Archaeologists (IFA) defines the archaeological archive as "all parts of the archaeological record, including the finds samples, and digital records as well as the written, drawn and photographic documentation" [5]. In defining the archive in this way the IFA have carefully avoided issues of appraisal and selection process. Museums select which of the artefacts they wish to retain, just as the archivists determine what archaeological information should be archived. Frequently, the decision about what to leave out is made according to the quality of the data. As a result many of the informal images are left behind, as the resource is not available ensure it meets archival standards.

Ideally, this archive, once deposited, can be accessed by anyone, whether involved in the excavation or not, and they will be able to interpret the results and where necessary incorporate this information into their own research. In reality, rarely are these records accessed and re-used, neither by archaeologists nor the general public. So even if an image makes it to the archive, it is not unusual for it to never be examined again.

For most archaeological investigations, reports are produced to meet requirements, not for mass consumption. As a result, much of this information is not broadly accessible beyond the discipline and the images remain inaccessible to the general public. Some excavations, particularly those of higher profile or that produce interesting results, will be published in books and increasingly, frequently on the web on specific archaeological and heritage websites. Archaeological sites of particular public interest may result in more popular publications and hopefully these are seen by a larger audience. However, this whole process typically takes years from beginning to end. 
For informal photography the process is very different. The selection and appraisal process is far less regimented. Instead of being part of a formal selection and appraisal process frequently images are lost because they are stored inappropriately or accidentally deleted or destroyed when a computer or camera fails. This is not to say that the photographers do not undertake a selection and appraisal process as they most certainly do, filtering for the images that they most desire.

If an image manages to not get deleted, destroyed or lost it will most likely end up on personal hard drives and shared with friends and family occasionally. This model has evolved in recent years, increasingly often but far from always images are shared on Flickr and Facebook and other social network sites where friends and family can see and comment on them at their leisure. In the case of Flickr, images are largely available to anyone who searches for them or follows the postings of a particular photographer or group. With the photographer and the audience adding comments and tags to the images their social values are enhanced as they act as a vehicle of communication as well as convey more information. Unlike formal dissemination, these photographs frequently end up online fairly quickly after they were taken, within days or weeks sometimes.

Frequently a further selection and appraisal process will occur. This occurs when other bloggers stumble on photographs on Flickr they find compelling or interesting and discuss those images they consider the most interesting. An excellent example of this comes from Colleen Morgan's blog, Middle Savagery [6], where she encourages her visitors to visit and post their archaeology images on the Flickr Group, Archaeology in Action [7], yet another form of informal filtering and selection.

The social role of the informal images is not unique to archaeology. Photography has served as medium of communication and memory sharing from the outset. It is worth exploring the different elements that can enhance the value of these images. The temporal nature of this photography can greatly impact its value, posting images of particularly interesting finds or contexts while it is still fresh in the minds of both the photographer and the subject can enable greater detail and explanation. Since much of archaeology is dependent on temporary short to medium term contracts away from home, these photographs frequently provide a vehicle for people to stay in touch with those they are no longer working or living with. This is not unique to archaeology nor is it necessarily always valuable to an archive. However, the opportunity to have this dialogue and engagement, even many years after the images were taken is perhaps worth exploring.

The formal dissemination and archiving of the archaeological photography does have one important strength over the informal dissemination and this is its link to the rest of the archaeological record. The need to deposit the entire archive in a single location means that the formal dissemination is contained together. The relationship between the photographs and the site record enables users to find information about the soil, related finds and other records. Detailed scaled drawings can be compared to understand better the relationships between the different archaeological elements. And where they are used in the final publication they are able to illustrate points made in the text. This is not to say that formal dissemination could not be accomplished using the wide variety of 
different dissemination tools available on the web but at present no examples of this could be found.

Engagement and dialogue, both with the formal and informal presentation of these images, provides a valuable opportunity for the general public to understand better how archaeology and archaeologists work. Currently, photo sharing websites accomplish this a great deal better than formal archives. Flickr is a well known site for finding images and frequently the first place people look for all sorts of photographs, not the heritage or digital archives. The informality of these sites should not be underestimated as well. Being able to look through different comments and engage with the photographer or others interested in the same sets of images allows for these images to have a life beyond the dusty shelves that the digital archive provides.

Additionally through Flickr Groups, for example, Archaeology in Action [8] it may be possible to accomplish a completely different task, previously unachievable; the capturing of archaeology across the globe on a day to day basis. Images from excavations provide a view of archaeology with only a limited regard to time or place. Many of these images go beyond documenting the archaeology and attempt to capture the conditions and the beauty of what to a field archaeologist is everyday.

Metadata is a largely uninteresting subject and the creation of it an unrewarding burden. Yet on social network sites, particularly Facebook and Flickr, they are just one more way to socialise using the photographs. Users and content creators can communicate about the images. Content creators can let visitors know about the location, time, purpose as well as information about those in the image. Visitors can ask questions about particular parts of the image or share personal experiences about the place in the image. They can provide feedback to the photographer about how they might improve the image the next time they take a picture. Ensuring that the user provided content is relevant and questions are answered requires a moderator [9].

It is clear that many heritage bodies are aware that they cannot sit idly by but they must find ways of getting involved. Flickr, through The Commons Project [10], has been working with heritage bodies to publish images from their collections since January 2008. This project hopes to not only shed light on the amazing images held within public archives but hopefully also to enhance their value. Looking through some of the images in the collection it is not always clear how well the public archives and the users are doing. One example of this is Brooklyn Museum's Early Lantern Slides [11]. On the one hand questions have been addressed by the Brooklyn museum archivists and there are loads of comments and associated tags, but a minority of the images have become littered with tags of limited or no value. This highlights a point raised from the experience of Wessex Archaeology when they started posting their images on Flickr [12], not only to moderate the postings and answer questions but to engage with discussion and ensure that you are part of the community, not simply sharing images.

It is not just heritage bodies but also commercial and academic units that have decided to post their formal images onto Flickr, for instance Wessex Archaeology [13] along with Michigan State University [14]. Wessex Archaeology has been taking a very active role on Flickr. Looking at their profile, they are not only sharing images but they 
are also using it to direct traffic to their main site. The Michigan State University profile is much newer, and as a result less developed. Although they share their photography, it appears that this is all that they have done so far.

\section{Working Shots as Part of the Archive}

What role do the informal images captured by site staff on their own cameras have to the archive as a whole? Working shots have an inherent value for demonstrating working practice and conditions on site. They can be put on websites and into reports and presentations. Occasionally these shots can even be useful in resolving difficult issues with the record [15]. Beyond these formal requirements they capture the archaeologist, their personalities and presence on site. Frequently these shots capture the camaraderie, in a field that values its professionals passion for their work. Surely this too is worth preserving.

So what information does the formal record encourage us to retain about working shots? Site photography at minimum will have the name of the site and the project number; this will be accompanied by the date and a description. Frequently the names of the archaeologists themselves are not captured. Interestingly this has been reflected in most of the images that appear in popular archaeology magazines, where rarely are the names of those excavating recorded.

When we consider the informal dissemination of these images, particularly on Facebook, the social recording of information inverts the priorities. This is likely to be in no small part because of the useful tools for tagging friends they provide. Thanks to them most images do have a name (frequently blocked by users' settings) but commonly fail to have both location and date. As a resul,t future archives will not be able to provenence these images effectively. So far Facebook has not embraced geotagging like some sites. Flickr fortunately does allow for geo-tagging as well as capturing the time from the camera itself and as result can show images according to their location. Ultimately the higher quality the data associated with these images the greater likelihood that they will complement future archives.

\section{Why Keep These Images?}

Is this information really that important? Well, for the history of archaeology it is. When we look at many of the historical archaeological photographs we have today, we frequently have very little information about who is in the photographs, aside from the most recognised of archaeologists. Many of the nameless faces involved have been forgotten. Their participation is absent from the record. But when looking back on the work of a particular archaeologist of regard or a relative, if there image is within the formal archive, it should be possible to identify them from the records. After all recording these extra little bits of information either in the formal or informal record, requires a limited amount of effort. The time has come to improve our recording of this type of information and to start looking for better tools to facilitate this.

It is obvious to say that both the informal and formal record would benefit from better recording; this is likely to always be true. Fortunately, for the formal record there are obligations in place to ensure that for its time the photographs should be effectively 
documented. No such obligations pertain to informal photography; therefore capturing the necessary information is dependent on the user and the added value they wish to give these images as well as on the information derived from viewers' questions and comments.

So how do we manage these images? To what extent can statutory organisations or employers control the informal dissemination? Sure an employer might be able to make a copyright claim on images taken on work time but to what end? Is it really to the benefit of archaeology to discourage people from posting images on the internet as long as they show archaeology and respect the privacy of their subjects? Surely the best approach is to embrace these images, assist in their effective documentation and encourage basic standards. By doing so we will ultimately ensure that others see these images and value our work. Controlling the dissemination of these images if it were possible would only do more harm than good. It is not about ownership or copyright of the images but about engaging with the public and encouraging them to engage with heritage.

\section{CONCLUSION}

If archaeology is going to benefit from the informal photography posted on social networking sites then it needs to engage with them. Not just by posting images but also through monitoring and selecting those images that are valuable. The sooner this is done the more information will be preserved with these images. Use of personal digital cameras on site is not likely to decrease. In fact, the more confident and skilled archaeologists are at taking photographs, the better all of site photography will be, whether it is formal or informal. And we need to encourage the collection of appropriate information that will not only be relevant in pure archival terms but will be socially relevant as well. As a discipline we should not discourage the informal dissemination of site photography over the internet. Instead, we need to embrace it and use these resources to share the work we are doing, potentially raising the profile of the work we do.

\section{References}

[1] ENGLISH HERITAGE. Archaeological projects recording manual Module 7 : Photography Section 7.0. [Unpublished], 2008.

[2] Ibid, Section 7.5.2.

[3] CONLON, V. M. Camera techniques in archaeology. London : John Baker; New York : St Martin's Press, 1973. pp.56-57.

[4] CRIPPS, P. Revelation : Appendix 4. Data modelling. English Heritage, 2003. pp 4-5.

[5] THE INSTITUE FOR ARCHAEOLOGISTS. Standards and guidance for the creation, compilation, transfer and deposition of archaeological archives., [published online October 2008]. http://www.archaeologists.net/modules/icontent/inPages/docs/codes/draftArchiv es2008.pdf [p.2 accessed 21 May 2009] 
[6] MIDDLE SAVAGERY Archaeology in action Update [published online April 2008] http://middlesavagery.wordpress.com/2008/04/26/archaeology-in-actionupdate/ [accessed 20 May 2009]

[7] Archaeology in action. Flickr. http://www.flickr.com/groups/archaeologyinaction/ [accessed 19 May 2009]

[8] Ibid.

[9] GOSKAR, T. Wessex Archaeology and Flickr : how we use Web 2.0. Culture24. December 2006.

http://www.culture24.org.uk/spliced/pictures/art41987.

[10] The Commons. Flickr [established January 2008]. http://www.flickr.com/commons/ [accessed 16 May 2009]

[11] Egyptian lantern slides - places. Flickr, Brooklyn Museum, May 2008. http://www.flickr.com/photos/brooklyn_museum/sets/72157605038624179/ [accessed 21 May 2009]

[12] GOSKAR, T. Wessex Archaeology and Flickr : how we use Web 2.0. Culture24. December 2006.

http://www.culture24.org.uk/spliced/pictures/art41987.

[13] Wessex Archaeology's photostream. Flickr, Wessex Archaeology, September 2006. http://www.flickr.com/photos/wessexarchaeology/ [accessed 22 May 2009]

[14] MSU Campus Archaeologist's Photostream Flickr, Michigan State University, April 2009. http://www.flickr.com/photos/34892027@,N05/ [accessed 22 May 2009]

[15] Using our Flickr photo galleries. April 2009. http://campusarch.msu.edu/CAPBlog/files/0c5e46c2e115fdd06ad861223d1f074 6-9.html [accessed 20 May 2009] 